Research Article

\title{
Energy-Optimized Pharmacophore Coupled Virtual Screening in the Discovery of Quorum Sensing Inhibitors of LasR Protein of Pseudomonas aeruginosa
}

\author{
Zulkar Naina, Sifat Bin Sayeda, Mohammad Minnatul Karima , Md. Ariful Islama, Utpal Kumar Adhikari ${ }^{{ }^{*}}$ \\ aDepartment of Biotechnology and Genetic Engineering, Islamic University, Faculty of Biological Sciences, Kushtia-7003, \\ Bangladesh. \\ bSchool of Medicine, Western Sydney University, Campbelltown, NSW-2560, Australia. \\ *Corresponding author: u.adhikari@westernsydney.edu.au
}

\begin{abstract}
Pseudomonas aeruginosa is an emerging opportunistic pathogen responsible for cystic fibrosis and nosocomial infections. In addition, empirical treatments are become inefficient due to their multipleantibiotic resistance and extensive colonizing ability. Quorum sensing (QS) plays a vital role in the regulation of virulence factors in P. aeruginosa. Attenuation of virulence by QS inhibition could be an alternative and effective approach to control infections. Therefore, we sought to discover new QS inhibitors (QSIs) against LasR receptor in P. aeruginosa using chemoinformatics. Initially, a structurebased high-throughput virtual screening was performed using the LasR active site that identified 61404 relevant molecules. E-pharmacophore (ADAHH) screening of these molecules rendered 72 QSI candidates. In standard-precision docking, only 7 compounds were found as potential QSIs due to their higher binding affinity to LasR receptor $(-7.53$ to $-10.32 \mathrm{kcal} / \mathrm{mol}$ compared to $-7.43 \mathrm{kcal} / \mathrm{mol}$ of native ligands). The ADMET properties of these compounds were suitable to be QSIs. Later, extra-precision docking and binding energy calculation suggested ZINC19765885 and ZINC72387263 as the most promising QSIs. The dynamic simulation of the docked complexes showed good binding stability and molecular interactions. The current study suggested that these two compounds could be used in $P$. aeruginosa QS inhibition to combat bacterial infections.
\end{abstract}

Keywords: Pseudomonas aeruginosa; Quorum sensing; Virtual screening; E-pharmacophore; Drug discovery.

\footnotetext{
Abbreviations

QS, Quorum sensing; QSI, quorum sensing inhibitor; VS, virtual screening; HTVS, high-throughput virtual screening; EF, enrichment factor; ROC, receiver operating characteristic; BEDROC, Boltzmann-enhanced discrimination of ROC; RIE, robust initial enhancement; AUAC, area under the accumulation curve; $\mathrm{GH}$, goodness of hit; SA, surface area; SASA, solvent accessible surface area; RMSD, root mean square deviation; RMSF, root mean square fluctuation; $\mathrm{SP}$, standard precision; $\mathrm{XP}$, extra precision; MW, molecular weight; MV, molecular volume; FOSA, hydrophobic component of the SASA; PISA, Pi component of the SASA; FISA, hydrophilic component of the SASA; WPSA, weakly polar component of the SASA; IP, Ionization potential; EA, electron affinity; HBD, hydrogen bond donor; HBA, hydrogen bond acceptor; PDB, protein data bank; MDS, molecular dynamic simulation; ADMET, absorption, distribution, metabolism, excretion, and toxicity; and MM-GBSA, molecular mechanics-generalized Born surface area.
} 


\section{Introduction}

During the past several decades, Pseudomonas aeruginosa has emerged as an important opportunistic human pathogen [1]. About $\sim 11 \%$ of hospital-acquired infections that contribute to a mortality rate of up to $61 \%$ are caused by the strains of $P$. aeruginosa [2,3]. It can cause cystic fibrosis (CF), urinary tract infections and burn infections, especially in immunocompromised patients [2,4]. In addition, P. aeruginosa becomes one of the most notorious pathogens due to its extensive colonizing ability, and its biofilm causes numerous other complications and treatment thereof [5]. For example, chronic lung infection in CF patients is caused by mucoid strains that grow biofilm [6]. In addition, the extensive biofilm formation with concomitant genetic plasticity is associated with a high incidence of antibiotic resistance [7,8]. As a result, existing antibiotics are losing control over $P$. aeruginosa and prompting scientists to look for new approaches to dealing with the infections $[8,9]$.

Quorum sensing (QS) regulates the expression of the genes responsible for pathogenesis, biofilm formation, and antibiotic resistance [10,11]. In P. aeruginosa, two QS systems (LasI/R and RhlI/R) regulate the expression of virulence and associated traits that act hierarchically [12,13]. The LasI/R system directs the synthesis of the 3-oxo-C12 homoserine lactone (HSL), protease, exotoxin A, elastase [12], while RhlI/R operates C4-HSL synthesis [14]. These molecules are natural ligands for LasR and RhlR receptors, respectively and activate virulence expression upon binding [15]. In addition, the HSL signaling system triggers a third P. aeruginosa quinolone signaling (PQS) system which also contributes to the expression of virulence factors, bacterial motility, and biofilm formation [16]. Thus, the entire QS cascade depends on the activation of LasI/R system. Therefore, deactivating this circuit would disintegrate the whole system which could prevent the pathogenesis of $P$. aeruginosa, as well as its biofilm formation and associated problems $[17,18]$.

QS inhibitors (QSIs) are chemical agents that can reduce microbial virulence by attenuating QS in bacteria. Several natural and synthetic compounds are known to act like QSI. For example, the most promising natural anti-QS compound reported to date is halogenated furanone from a marine alga Delisea pulchra [18]. Some others natural QSIs are patulin and penicillic acid from Penicillium species [19], ajoene, a sulfur-rich molecule from garlic [20] and ellagic acid derivatives from Terminalia chebula Retz [21]. However, natural QSIs generally have a narrow spectrum of activity and limited clinical value [22]. In addition, current halogenated furanones are too reactive, and therefore presumably too toxic to treat bacterial infections in humans. Moreover, attenuation of a single species within a polymicrobial community (e.g., wound sites) is not possible due to their functional non-specificity [23]. Like anti-QS phytoconstituents, biochemical compounds are also known for anti-QS functionality. For instance, subtilosin may inhibit E. coli QS signals to control biofilm formation [24]. However, their antimicrobial effects are also limited [25].

In recent years, chemoinformatics approaches such as structure-based virtual screening (SBVS) have been successfully used in computational drug discovery, significantly reducing the time, effort, and expense of traditional methods [26,27]. Virtual screening (VS) is a computational method that searches 
compound libraries to identify potential molecules that can efficiently bind to a drug target [27]. The increasing availability of structural data and the affordability of high-performance computing platforms have broadened the applicability of this method [28]. For example, the crystal structure of QS receptor (i.e., LasR) could be used as a target to discover potential QSIs through SBVS methods. Therefore, it is promising for the discovery of drugs against pathogens and in particular for the identification of new QSIs [29]. Recently, some success has been achieved in the determination of inhibitors of human carbonic anhydrase II [30], human protein tyrosine phosphatase 1B [31], and DNA gyrase [32], G-protein-coupled receptors, apoptosis regulators (Bcl-2, Hsp90) [29,33]. The in silico discovery of QSIs against LasR receptor has already been studied [14,15]. However, their study was conducted on a small number of chemical compounds whose validation was not attempted by a joint pharmacophore mapping and molecular dynamics simulation (MDS) to further clarify their message.

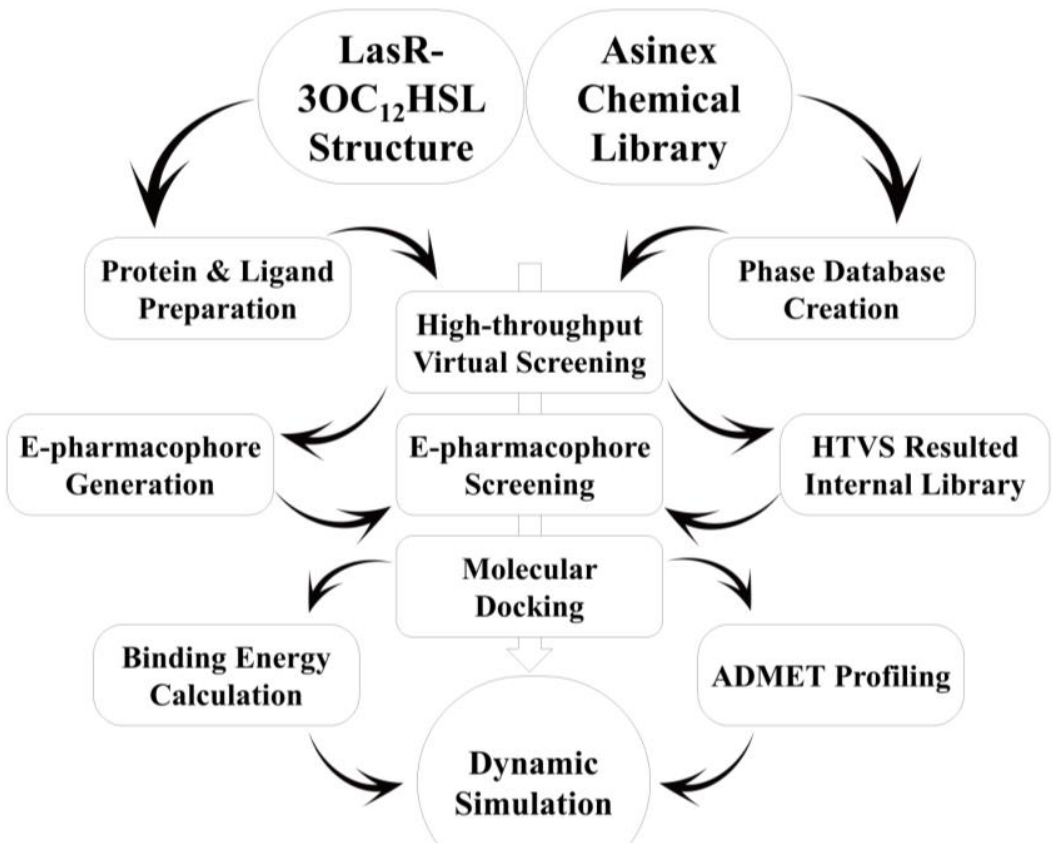

Figure 1: Experimental workflow for designing of QS inhibitor against P. aeruginosa LasR protein.

In this study, chemoinformatics approaches were applied to find potential QSIs against LasR protein and evaluating their efficiency in silico. The selection of potential QSIs was executed in three steps: (i) highthroughput virtual screening to opt-out the irrelevant compounds, (ii) pharmacophore screening to identify molecules suitable for LasR binding site, and (iii) binding affinity dependent selection through standard precision molecular docking (Figure 1). The Asinex Chemical Library was used as screening database. Potential ligands filtered through three consecutive screening were subjected to ADMET analysis and re-docking with extra-precision mode along with binding energy calculation. Finally, best ligands were subjected to MDS studies that are superior in both docking modes and have good ADMET profile. 


\section{Experimental Workflow}

The entire computational studies were carried out in Windows 10 Professional operating environment installed on Sony VAIO Fit 15A laptop equipped with Intel (R) Core i7 (4th $\mathrm{Gen}) 4500 \mathrm{U} / 1.8 \mathrm{GHz}$ processor (Turbo Boost up to 3.0GHz), 8GB DDR3L RAM, 1TB ROM, and 2GB NVIDIA GeForce GT 735M graphics. All chemoinformatics analyses were performed in Maestro v11.8 (Schrödinger, LLC, New York, NY) except for the molecular dynamics simulation which was executed with YASARA Dynamics v17.8 (by Dr. Elmar Krieger, YASARA Biosciences).

\subsection{Retrieval and preparation of protein structure}

The crystal structure of the LasR protein along with its native ligand was downloaded from the Protein Data Bank (PDB) (https://www.rcsb.org/). Protein structure obtained from the PDB was not suitable in its native state for molecular docking [14]. Therefore, the protein structure was optimized with Schrödinger's Protein Preparation Wizard for proper bond orders, the addition of hydrogen atoms and missing residues, correction of formal charges to the hetero groups, and generation of tautomer/ionization states at physiologic $\mathrm{pH}$ (7.0) [34]. The water molecules present in most crystal protein structure [35]. Therefore, water molecules beyond $5 \AA$ distance around the protein's natural ligand were deleted. Further, the protein structure was minimized using until the heavy atoms converged to the RMSD of $0.30 \AA$ using OPLS_2005 force field [36] which uses different torsional parameters to reproduce the conformational energetics to support organic functionality [36,37]. Finally, the interaction of the co-crystal ligand with the active site residues of LasR protein was analyzed and cross-checked with PDBsum server (http://www.ebi.ac.uk/pdbsum).

\subsection{Receptor grid generation}

The generation of a defined grid-box on the protein's active site is a prerequisite for prospective docking studies. Firstly, the co-crystallized ligand was dissected from the binding site of LasR receptor. The Glide v8.1 module [38] was applied to the receptor protein to define a grid-box around the co-crystallized ligand which will allow docking into the active site [39]. Thus, the active site was defined as an enclosing box $(72 \times 72 \times 72 \AA)$ at the centroid of the co-crystallized ligand within which a smaller secondary box $(27 \times 27 \times 27 \AA)$ was created and centered around the potential binding site of interest. The scaling of van der Waals radii was set to defaults where scaling factor was $1.0 \AA$ and the partial atomic charge was less than 0.25 as a cutoff. No rotatable groups were selected and no constraints were defined.

\subsection{Creation of phase database}

The compounds in the range of $\mathrm{pH}$ 6-8 were collected from Asinex Chemical Library (Moscow, Russia) through the ZINC database (http:/ / zinc12.docking.org) [40]. The 3-dimensional structural coordinates of all molecules were retrieved in SDF format. The Phase module (Maestro 11.8, Schrödinger) was used to create a ligand database of these molecules. The LigPrep and Epik v4.6 [41] were applied to expand protonation and tautomeric states at $\mathrm{pH} 7.0 \pm 2.0$ for each molecule. The chirality of ligands was determined from their three-dimensional structures. In addition, QikProp properties were generated based on which Lipinski's Rule of $5{ }^{[42]}$ was applied to filter potential ligands. Also, reactive functional groups were skipped which may cause false positive in high-throughput screening [43,44]. 


\subsection{High-throughput virtual screening}

In drug or inhibitor design, the structure-based high-throughput virtual screening (HTVS) have been applied successfully to identify potential drug candidates [45]. It facilitates rapid scanning of a large ligand database consisting of millions of compounds to determine potential molecules that can fit into and interact with the protein's active site perfectly [46]. We used HTVS protocol of Glide module [38] for ligandbased virtual screening with flexible ligand sampling. For rendering a Glide score, the number of maximum atoms and rotatable bonds in a ligand was set to 500 and 100, respectively. The van der Waals radii scaling was set to default (i.e., scaling factor $=0.80$ and partial charge cutoff $=0.15$ ) and post-docking minimization was also performed with 5 poses maximum for each ligand.

\subsection{Development of e-pharmacophore hypothesis}

The generation of energy-optimized pharmacophore (e-pharmacophore) [47] is important for virtual screening to be fast and efficient as it achieves the benefits of both ligand- and structure-based approaches [48]. The canonical SMILES of the dissected co-crystal ligand of LasR protein was submitted to PubChem database [49] for similarity searching and two most structurally similar compounds were collected. Further, three ligands (including native ligand) were prepared using Schrödinger's LigPrep module and docked into the LasR protein using Glide XP docking [50]. The 'pose-viewer' files of these three docked complexes were then used for e-pharmacophore modeling with Phase module [51] where a default set of six chemical features: hydrogen bond acceptor (A), hydrogen bond donor (D), hydrophobic $(\mathrm{H})$, negative ionizable $(\mathrm{N})$, positive ionizable $(\mathrm{P})$, and aromatic ring $(\mathrm{R})$ were employed [48]. For precise matching, pharmacophoric sites with fitness score lower than -0.5 were rejected [34,52]. In this study, the generated three different e-pharmacophores were merged together to generate a common epharmacophore model.

\subsection{Validation of e-pharmacophore mapping}

The authenticity of a pharmacophore to identify potential ligands from a bulk of anonymous compounds needs to be validated [53]. Therefore, some important measures, i.e., enrichment factor (EF), receiver operating characteristic (ROC) [54], Boltzmann-enhanced discrimination of ROC (BEDROC) [55], robust initial enhancement (RIE) [56], goodness of hit (GH) [48] were taken into account to determine the performance of the generated e-pharmacophore [57]. An internal compound library $(n=1003)$ was created by combining three ligands as actives and Schrodinger's 1000 drug-like compounds as decoys [38,58]. The common e-pharmacophore model was then screened against the internal library. The incurred results were then assessed with EF, ROC value, BEDROC $(a=20)$ metrics, RIE, and GH score which are often used to standardize the VS protocol [34,59]. The following equations were used to calculate the EF and GH score:

Enrichment factor $(E F)=\frac{H_{A}}{H_{T}} \times \frac{D}{A}$ 


$$
\text { Goodness of hit }(G H)=\frac{H_{A}\left(3 A+H_{T}\right)}{4 \times A \times H_{T}} \times\left(1-\frac{H_{T}-H_{A}}{D-A}\right)
$$

Where, $\mathrm{EF}=$ enrichment factor, $\mathrm{GH}=$ goodness of hit, $\mathrm{D}=$ total compounds in the internal library, $\mathrm{A}=$ number of actives in the library, $\mathrm{H}_{\mathrm{T}}=$ number of total hits, and $\mathrm{H}_{\mathrm{A}}=$ the number of active hits.

\subsection{E-pharmacophore database screening}

Based on the structural features embodied in the validated e-pharmacophore, the Phase module was used for shape-based similarity search [60]. Pharmacophore screening of compounds was performed against compound hits obtained from the HTVS to identify the compounds which also possess important pharmacophore sites. The screening molecules required to match a minimum of 4 sites among the total 5 sites to be selected. As a balance between stringent and loose-fitting alignment, the distance matching tolerance was set to $2.0 \AA$ [61]. The existing conformers and match rejection criteria were set to defaults. The hits obtained from the pharmacophore screening were exported as a library of potential LasR inhibitors.

\subsection{Molecular docking studies}

Docking is the binding orientation of small molecules to their protein targets in order to predict the affinity and activity of the small molecules, hence, plays an important role in the drug design [62]. The Glide v8.1 module was used to estimate the binding interactions between the receptor protein and ligands [38]. Briefly, the potential ligands (including the native ligand as positive control) filtered through the pharmacophore screening were subjected to energy minimization using the LigPrep v2.2 module of Schrödinger. Then the ligands were subjected to molecular docking against the binding site of LasR to find the best QSIs possible. The molecular docking was performed in both standard precision (SP) and extra precision (XP) modes. The interactions between the receptor and ligand molecules were calculated as Glide scores, i.e., G-scores, based on the following equation:

$$
\text { Glide score }=0.065 \times \mathrm{vdW}+0.130 \times \text { Coul }+ \text { Lipo }+ \text { Hbond }+ \text { Metal }+ \text { BuryP }+ \text { RotB }+ \text { Site }
$$

Where, $\mathrm{vdW}=$ van der Waals energy, Coul $=$ Coulomb energy, Lipo $=$ lipophilic term derived from hydrophobic grid potential, Hbond = hydrogen-bonding term, Metal = metal-binding term, BuryP = penalty for buried polar groups, $\operatorname{Rot} \mathrm{B}=$ penalty for freezing rotatable bonds, and Site = polar interactions present in the active site.

\subsection{Prime MM-GBSA calculation}

Molecular mechanics-generalized Born surface area (MM-GBSA) method was used as a post-docking validation tool [63]. The binding energy calculated by MM-GBSA shows a reasonably good correlation between the predicted and the experimental binding affinity [63]. Besides, the post-docking binding energy calculation is more efficient when the ranking of congeneric inhibitors is emphasized [64,65]. The Prime/MM-GBSA method (Maestro v11.8, Schrodinger) combines OPLS molecular mechanics energies (EMM), a VSGB solvation model for polar solvation (GSGB), and a nonpolar solvation term (GNP) 
composed of the nonpolar solvent-accessible surface area (SASA) and van der Waals interactions [66]. Based on the docked complex, it calculates the binding free energy $\left(\Delta \mathrm{G}_{\mathrm{BIND}}\right)$ of each ligand using the following equation [64,67].

$$
\Delta \mathrm{G}_{\mathrm{BIND}}=\Delta \mathrm{E}_{\mathrm{MM}}+\Delta \mathrm{G}_{\mathrm{SOLV}}+\Delta \mathrm{G}_{\mathrm{SA}}
$$

Where, $\Delta \mathrm{E}_{\mathrm{MM}}=$ the energy difference between the complex structure and the sum of the energies of the protein with and without ligand, $\Delta G_{S O L V}=$ difference in the GBSA solvation energy of the complex and the sum of the solvation energies for the ligand and unliganded protein, and $\Delta \mathrm{G}_{\mathrm{SA}}=$ difference in the surface area energy for the complex and the sum of the surface area energies for the ligand and uncomplexed protein.

Initially, docked poses were minimized through local optimization followed by the measuring of the complex's energies using OPLS_2005 force field [68]. The simulations were carried out by GBSA continuum solvent model [67]. Prime uses a surface generalized Born (SGB) model employing a Gaussian surface instead of van der Waals surface for better representation of a solvent accessible surface area [67].

\subsection{Ligand-based ADME/Tox prediction}

Drug-like molecules exhibit favorable ADMET (absorption, distribution, metabolism, excretion, and toxicity) parameters [68]. Therefore, we predicted the drug-likeness and pharmacokinetic profiles of the potential QSI molecules with Schrodinger's QikProp v5.8 module [69]. It provides a range for comparing a particular molecule's properties with those of $95 \%$ of known drugs [68]. All the compounds were neutralized before QikProp operation. The program was carried out in normal mode, and predicted 44 properties for the molecules, consisting of principal descriptors and physicochemical properties with a detailed analysis of the $\log$ P (Octanol/Water), QP\%, and log HERG. It also evaluates the acceptability of the compounds based on Lipinski's rule of 5 [42], which are essential for rational drug design [68].

\subsection{Molecular dynamic simulation}

The molecular dynamics simulation (MDS) is essential to evaluate the stability of the receptor-ligand complex [70] and to understand how they would behave in a pre-defined environment through atomic movements [63]. Therefore, we performed the MDS of the two most promising ligand-receptor complexes using YASARA Dynamics with AMBER14 force field [71,72]. MDS studies were carried out within a predefined simulation cell solvated with TIP3P water (density: $0.997 \mathrm{~g} / \mathrm{L}$ ). The simulations were executed at physiological conditions $(298 \mathrm{~K}, \mathrm{pH} 7.4,0.9 \% \mathrm{NaCl})$ for $30 \mathrm{~ns}$ time period. Prior to the simulations, the complexes were cleaned and optimized for hydrogen-bond networks and cut-off radius was set to $8.0 \AA$ A. Timestep of 2.50 fs was used and simulation snapshots were captured at every 250 ps. Finally, the trajectories were analyzed for the stability of the complexes in terms of the root mean square deviation (RMSD) and root mean square fluctuation (RMSF) for backbone and side-chain, respectively. Since hydrogen bonds are important for stable and precise binding [73,74], the number of hydrogen bond present in the average simulation complex was also analyzed. Furthermore, the surface area of the LasR 
protein was also calculated with and without binding to its ligand to understand the occupancy of the potential QSI candidates to the receptor cavity.

\section{Results and Discussion}

\subsection{The LasR protein and binding site analysis}

The P. aeruginosa QS receptor, LasR, is a transcriptional activator protein [75]. The crystal structure of LasR protein with $1.4 \AA$ resolution (PDB: 3IX3) was retrieved from PDB database [76]. The protein structure had bound its native ligand, N-3-oxo-dodecanoyl-L-homoserine lactone $\left(3 \mathrm{OC}_{12}\right.$-HSL). The active site of the LasR protein is defined by the residues present around $4 \AA$ region of the co-crystal ligand. The analysis of ligand binding interaction revealed that active site residues, i.e., Leu36, Try47, Try56, Trp60, Tyr64, Asp73, Thr75, Val76, Cys79, Trp88, Tyr93, Phe101, Ala105, Leu110, Gly126, and Ser129 were involved in all non-bonded interactions in which only five residues, namely, Tyr56, Trp60, Asp73, and Ser129 contributed in the hydrogen bond (HB) networking. The precise and stable binding of the ligands to the active site depends on the HBs [73,74] as it provides specificity and directionality in the interactions [77]. Therefore, a potential QSI should be able to produce good HBs with the active sites.

\subsection{Phase database and high-throughput virtual screening}

Phase database facilitates rapid screening [60]. We created a Phase database consisting of total 117106 compounds ( $\mathrm{pH}$ 6-8) included in the Asinex Chemical Library with Phase module [60]. The LigPrep program generated a total of 318452 conformational states from input 117106 structures. Among all the generated states, 218682 have passed both Lipinski filter and reactive filter, hence, considered for the Phase database. The result suggests a large database was created with a high number of compounds in their most desirable states possible. For high-throughput virtual screening (HTVS), the molecules from Phase database were docked into the generated grid-box area in the LasR active site which resulted in the identification of 61404 compounds as the potential match for the binding cavity.

\subsection{Energy-optimized pharmacophore mapping}

E-pharmacophores are generated by optimized energies from interactions mapped onto the atom center [34]. The generation of multiple hypotheses is required to identify the best pharmacophore model that is able to render a potential outcome ${ }^{[78]}$. Also, molecules shared structure similarity also possess similar activity [79]. Therefore, two structurally similar compounds, i.e., CID9795780 (3-oxo-N-(2-oxooxolan-3yl)undecanamide) and CID9882952 (4,4-Difluoro-3-oxo-N-(2-oxooxolan-3-yl)decanamide), were retrieved using the native ligand. Both native ligand and CID9795780 provided the same five featured model (ADAHH). On the other hand, CID9882952 generated a hypothesis with four pharmacophoric features (ADAH) as shown in Figure 2a. A common e-pharmacophore hypothesis (ADAHH) was generated by merging these three hypotheses (Figure 2b). The hydrophobic site H5 lies on the C14 atom, $\mathrm{H} 4$ on $\mathrm{C} 13$ atom of the ligand. The first acceptor, A1, lies on C-O-C (O6 atom), and the other acceptor, $\mathrm{A} 2$, on the $\mathrm{C}=\mathrm{O}$ (O9 atom) group of the crystal ligand while donor, D3 lies on N-H (N12 atom) group of the merged hypothesis. 
(a)
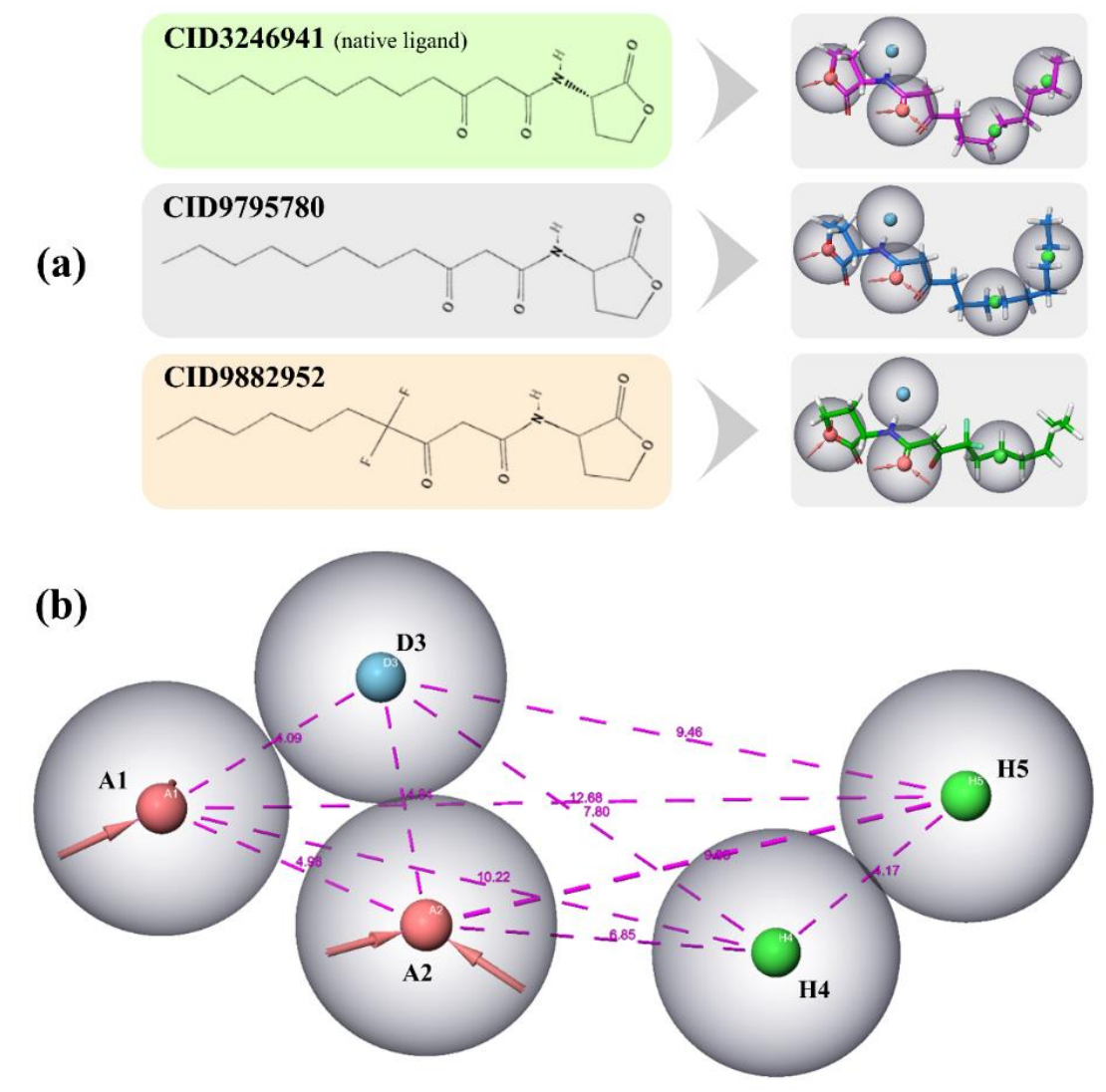

Figure 2: The mapping of e-pharmacophore hypothesis. The figure includes (a) three individual epharmacophore models viz., CID3246941/native ligand (pink), CID9795780/(3-oxo-N-(2-oxooxolan-3yl)undecanamide (blue), and CID9882952/4,4-Difluoro-3-oxo-N-(2-oxooxolan-3-yl)decanamide (green), (b) a merged common hypothesis containing five pharmacophore sites viz., two proton acceptor (A1 and A2), a single proton donor (D3) and two hydrophobic sites (H4 and H5).

\subsection{E-pharmacophore validation and database screening}

The validation of the pharmacophore is essential to check the model's efficiency in retrieving the actives and early ranking [78]. For this purpose, different enrichment parameters were calculated that are presented in Table 1. EF is the quality of a pharmacophore to differentiate between actives and decoys which is calculated based on the number of identified known actives from a fraction of database screened [80]. We emphasized on the EF (1\%) since it measures the enrichment score for the top $1 \%$ of the decoys screened [52]. The EF (1\%) and GH score of the generated pharmacophore were 33.53 and 0.62 , respectively, which is able to identify actives from a large dataset of compounds [48]. The ROC value corresponds to the position of actives to the orderly ranked compounds that are linearly arranged among the defined internal library [34]. The ROC value ranged in between 0 to 1 where $\geq 0.7$ is considered as a suitable execution measuring value [55]. So, the ROC value of 0.99 in our study is outstanding for the selection of active molecules. In addition, the \% screen plot and ROC plot revealed the sensitivity and specificity for active molecule recognition. 
The BEDROC metrics measure the early recognition of actives from the database and ranges from $0-1$ score [78]. We considered $a=20.0$ for BEDROC metric which entails that $80 \%$ of the BEDROC results comes from the first $8 \%$ of the ranked molecules [55]. Therefore, a substantial BEDROC $(a=20.0)$ value of 0.788 suggested the early detection of active compounds from the database. All these enrichment results suggest that the generated e-pharmacophore was satisfactory in retrieving the actives from a large database of molecules.

Table 1: Enrichment matrices for energy-optimized pharmacophore validation

\begin{tabular}{ll}
\hline Enrichment matrices & Values \\
\hline NA $(1 \%, 2 \%, 5 \%, 10 \%, 20 \%)$ & $1,3,3,3,3$ \\
$\%$ A $(1 \%, 2 \%, 5 \%, 10 \%, 20 \%)$ & $33.3,100,100,100,100$ \\
EF $(1 \%, 2 \%, 5 \%, 10 \%, 20 \%)$ & $34,34,20,10,5$ \\
ROC & 0.99 \\
BEDROC $(\mathrm{a}=20)$ & 0.788 \\
AUAC & 0.99 \\
RIE & 15.30 \\
GH & 0.623 \\
\hline
\end{tabular}

${ }^{*} \mathrm{EF}$, enrichment factor; \%A, percentage of actives; NA, number of actives; ROC, receiver operating characteristic; BEDROC, Boltzmannenhanced discrimination ROC; AUAC, area under the accumulation curve; RIE, robust initial enhancement; GH, goodness of hit

Therefore, the high EF1\% (33.53) value with remarkable ROC (0.99), AUAC (0.99), RIE (15.30), and GH (0.623) scores indicated that the pharmacophore model generated in this study will be able to identify the potential QSI compounds from the compound library. Finally, the pharmacophore was used against the HTVS filtered 61404 compounds using the Phase module [60]. Pharmacophore screening resulted in the generation of 72 molecules having similar pharmacophore features.

\subsection{Molecular docking studies}

After the pharmacophore-based screening, potential 72 hit compounds were subjected to molecular docking. Initially received 72 hit compounds were docked into the active site of LasR receptor using standard-precision (SP) mode which ranked molecules based on docking scores. The SP docking score of native ligand ( $\mathrm{G}=-7.43)$ was used as a cutoff, and the score below -7.5 was ranked as highly active and above -7.5 as low active compounds. In this process, only 7 molecules, i.e., ZINC19765885, ZINC19453811, ZINC72360552, ZINC19717706, ZINC19291753, ZINC72387263, and ZINC19291739 (Figure 3) with docking score below -7.5 were identified as potential LasR inhibitors. These potential QSIs were further undergone extra-precision $(\mathrm{XP})$ docking to critically analyze the favorable interactions between the ligand and the protein. We found only two compounds (ZINC19765885 and ZINC72387263) having XP score less than that of the native ligand as provided in Table 2. 


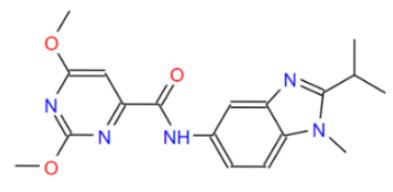

ZINC19453811

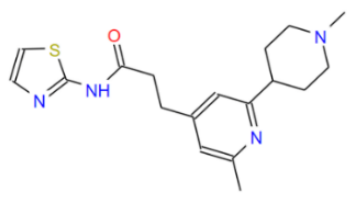

ZINC72360552
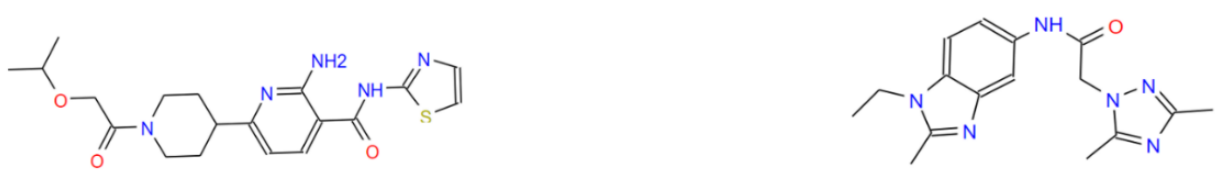

ZINC19765885

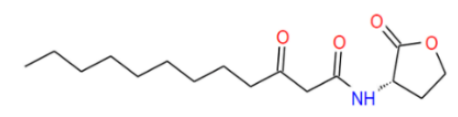

ZINC19717706

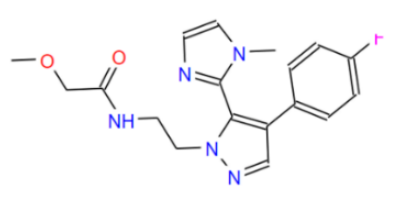

NATIVE LIGAND

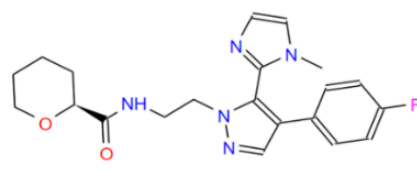

ZINC19291753

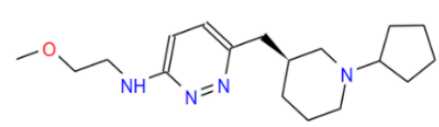

ZINC19291739

ZINC72387263

Figure 3: Two-dimensional (2D) structures of native ligand and seven potential hit molecules. The 2D structure of LasR native ligand is placed at the center for comparison with hit compounds.

Table 2: Molecular docking studies of LasR protein complexed with potential hit ligands.

\begin{tabular}{llllll}
\hline Compounds & $\begin{array}{l}\text { SP docking } \\
\text { (G-score) }\end{array}$ & $\begin{array}{l}\text { XP docking } \\
\text { (G-score) }\end{array}$ & $\begin{array}{l}\text { H-bond } \\
\text { (n) }\end{array}$ & $\begin{array}{l}\text { NB-contact } \\
\text { (n) }\end{array}$ & $\begin{array}{l}\text { Residues involved in } \\
\text { hydrogen bonds }\end{array}$ \\
\hline Native & -07.43 & -10.07 & 4 & 56 & Tyr56, Trp60, Asp73,Ser129 \\
Ligand & & & & & \\
Zinc19765885 & -10.32 & -10.37 & 4 & 92 & Trp56, Asp73, Thr75, Ser129 \\
Zinc19453811 & -08.38 & -07.51 & 2 & 72 & Trp60, Ser129 \\
Zinc72360552 & -09.40 & -08.76 & 1 & 56 & Ser129 \\
Zinc19717706 & -07.53 & -05.96 & 0 & 56 & - \\
Zinc19291753 & -08.65 & -07.50 & 4 & 72 & Tyr56, Asp73, Ser129 \\
Zinc72387263 & -08.17 & -10.15 & 1 & 64 & Asp73 \\
Zinc19291739 & -08.94 & -07.68 & 3 & 86 & Tyr56, Asp73, Ser129 \\
\hline
\end{tabular}

*G-score, docking score by Glide module; H-bond, number of hydrogen bonds; and NB-contact, number of non-bonded contacts.

The dock complexes from the XP docking were analyzed for protein-ligand interaction as given in Table 2 and Figure 4. Interestingly, a similar pattern in hydrogen bond (HB) interactions was observed for both Zinc19765885 and native ligand. The residues involved in the HB interactions were Trp56, Asp73, Thr75 
and Ser129 for ZINC19765885 and Tyr56, Trp60, Asp73, and Ser129 for the native ligand. Therefore, they share 3 out of 4 active site residues to produce HB interactions. Importantly, ZINC19765885 showed more interactions than the native ligand. For instance, the ZINC19765885 was involved with 92 non-bonded contacts while native ligand provided 56 non-bonded interactions. The ZINC19765885 also showed two $\Pi-\Pi$ stacking interaction with Trp88 and Phe101 residues. Despite the high binding affinity, however, the Zinc72387263 showed a single HB interaction with catalytic Asp73 of the active site and total of 64 nonbonded interactions. It also formed a salt-bridge with the Asp73. The detailed interactions between the LasR protein and prospective ligands were illustrated in Figure 4.

(a)

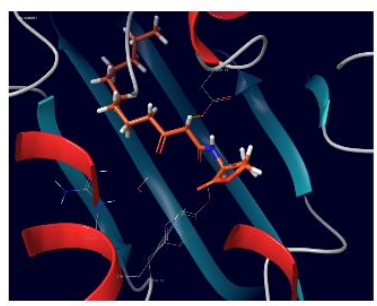

(b)

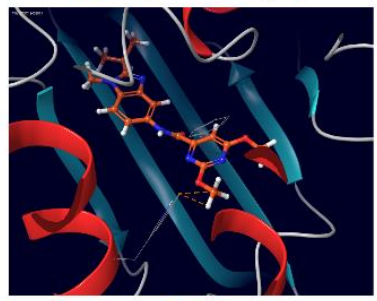

(c)

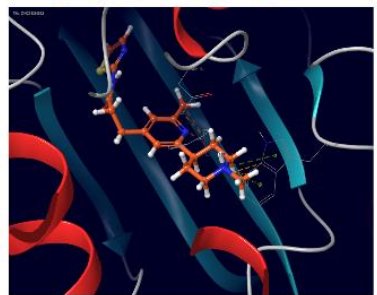

(d)

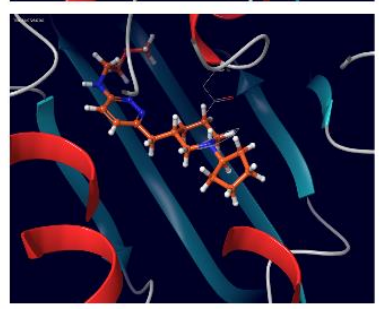

Charged (negative) Charged (positive) Glycine Hydrophobic Metal

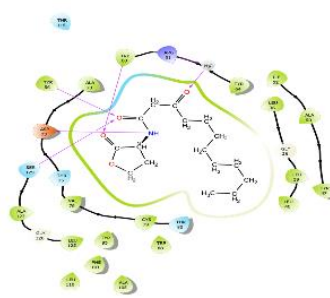

(e)
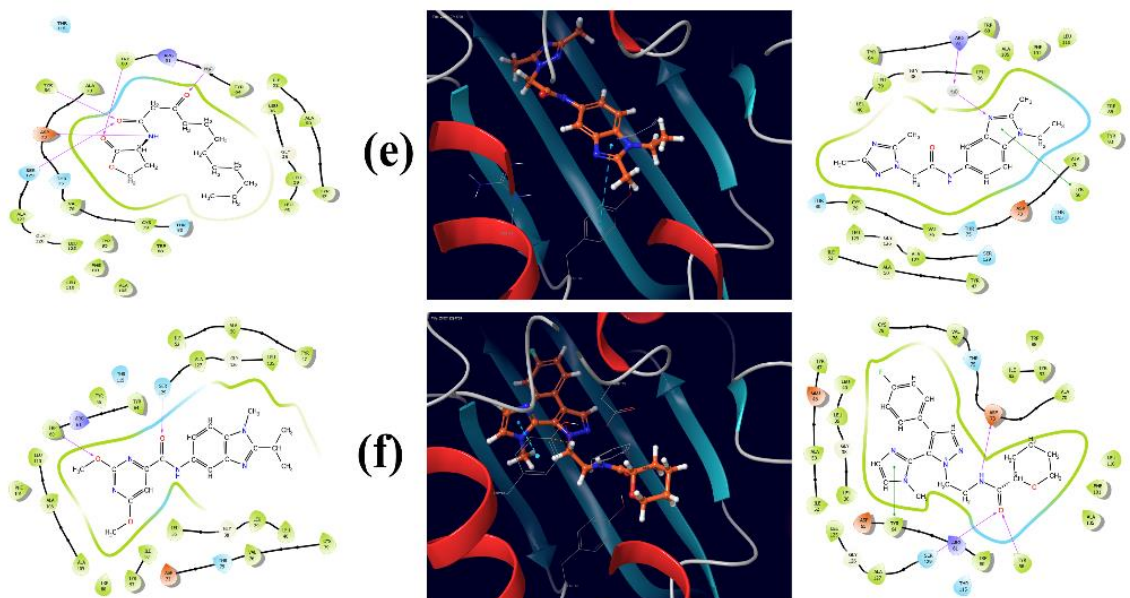

(f)
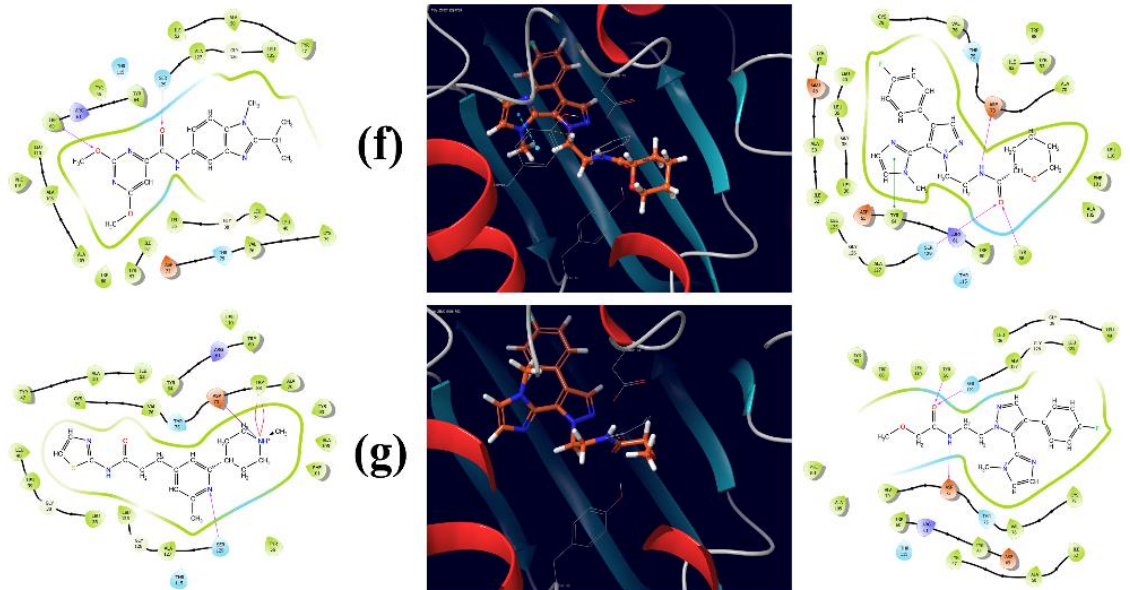

(g)
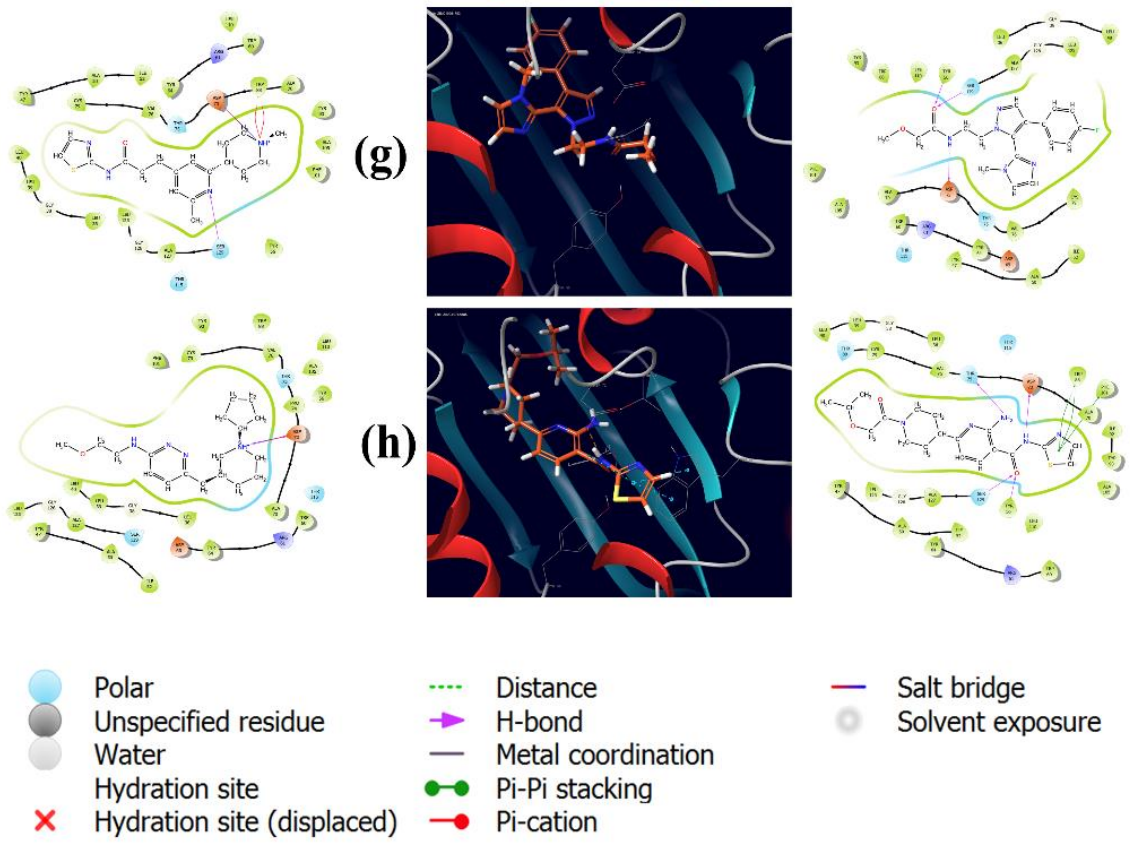

$\begin{array}{ll}\cdots & \text { Distance } \\ \rightarrow \quad & \text { H-bond } \\ -\quad \text { Metal coordination } \\ \rightarrow \quad \text { Pi-Pi stacking } \\ \longrightarrow \quad \text { Pi-cation }\end{array}$

- Salt bridge Solvent exposure

Figure 4: The molecular interactions between protein and potential QSI hits. The protein-ligand complexes from the XP docking showing: (a) native ligand interacted with Tyr56, Trp60, Asp73, and Ser129 residues through HB interactions (b) ZINC19453811 provided HB with Trp60 and Ser129 residues (c) ZINC72360552 showed a single HB interaction with Ser129 residue, (d) ZINC72387263 shared HB 
with Asp73 and Leu125 residues (e) ZINC19717706 produced no HB interactions, (f) ZINC19291739 interacted by HB with Tyr56, Asp73, and Ser129 residues, (g) ZINC19291753 shared HB with Tyr56, Asp73 and Ser129 residues, and (h) ZINC19765885 produced HB networking with residue Trp56, Asp73, Thr75 and Ser129 from active site of the LasR protein.

\subsection{Molecular descriptor and ADMET properties}

Due to poor ADMET profile, about $\sim 40 \%$ of drug candidates cannot pass the clinical trials [78]. The principal descriptors and ADMET properties were calculated by Schrodinger's QikProp v5.8 module. The ADMET profile of the seven potential compounds is provided in Table 3. All selected compounds

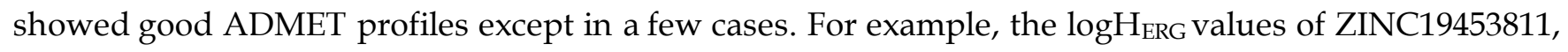
ZINC72360552, ZINC19717706, and ZINC72387263 were -5.39,-6.29, -5.20, and -5.43, respectively, which were in the concerning range. Furthermore, $\operatorname{logBB}$ value of the ZINC19765885 was -1.28 which is a bit lower than the lower acceptable range. Importantly, no compound violated Lipinski's rule of 5. Besides, a single violation of Rule of 3 was observed for ZINC72360552 which is in the acceptable range though (Table 3) [81].

Table 3: The ADMET properties of the proposed lead molecules.

\begin{tabular}{|c|c|c|c|c|c|c|c|c|}
\hline $\begin{array}{l}\text { ADMET } \\
\text { Properties }\end{array}$ & $\begin{array}{l}\text { ZINC } \\
19765885 \\
\end{array}$ & $\begin{array}{l}\text { ZINC } \\
19453811 \\
\end{array}$ & $\begin{array}{l}\text { ZINC } \\
72360552 \\
\end{array}$ & $\begin{array}{l}\text { ZINC } \\
19717706 \\
\end{array}$ & $\begin{array}{l}\text { ZINC } \\
19291753 \\
\end{array}$ & $\begin{array}{l}\text { ZINC } \\
72387263 \\
\end{array}$ & $\begin{array}{l}\text { ZINC } \\
19291739 \\
\end{array}$ & $\begin{array}{l}\text { Optimal Range } \\
\text { (in 95\% drugs) }\end{array}$ \\
\hline Polrz & 42.43 & 37.73 & 39.62 & 35.23 & 37.36 & 35.29 & 42.94 & 13.0 to 70.0 \\
\hline $\log \mathrm{P}_{\mathrm{C} 16}$ & 13.19 & 10.81 & 11.34 & 9.94 & 10.65 & 9.62 & 11.81 & 4.0 to 18.0 \\
\hline $\log P_{\text {oct }}$ & 23.58 & 16.96 & 18.14 & 16.69 & 18.46 & 15.83 & 20.22 & 8.0 to 35.0 \\
\hline $\log P_{w}$ & 16.75 & 9.31 & 10.49 & 9.76 & 12.20 & 8.29 & 12.47 & 4.0 to 45.0 \\
\hline $\log \mathrm{P}_{\mathrm{o} / \mathrm{w}}$ & 1.98 & 3.45 & 2.94 & 2.68 & 2.86 & 2.91 & 3.63 & -2.0 to 6.5 \\
\hline $\log S$ & -3.82 & -5.03 & -4.23 & -4.64 & -3.55 & -3.31 & -4.89 & -6.5 to 0.5 \\
\hline CIlogS & -3.81 & -4.95 & -3.08 & -3.84 & -4.13 & -2.56 & -4.97 & -6.5 to 0.5 \\
\hline $\log \mathrm{H}_{\mathrm{ERG}}$ & -4.03 & -5.39 & -6.29 & -5.20 & -3.87 & -5.43 & -4.29 & Concern below -5 \\
\hline PCaco & 172.51 & 1484.10 & 419.32 & 986.13 & 1608.12 & 857.76 & 1695.18 & $<25=$ poor $;>500=$ great \\
\hline $\log B B$ & -1.28 & -0.62 & -0.025 & -0.75 & -0.20 & 0.13 & -0.17 & -3.0 to 1.2 \\
\hline PMDCK & 254.07 & 758.02 & 362.88 & 487.29 & 2479.03 & 463.67 & 2541.19 & $<25=$ poor $;>500=$ great \\
\hline $\log K_{p}$ & -3.23 & -2.04 & -4.12 & -2.65 & -1.29 & -3.66 & -1.35 & -8.0 to -1.0 \\
\hline $\log \mathrm{K}_{\mathrm{hsa}}$ & -0.30 & 0.32 & 0.24 & 0.08 & -0.21 & 0.18 & 0.15 & -1.5 to 1.5 \\
\hline pHOA & 78.57 & 100.00 & 91.08 & 96.21 & 100.00 & 96.47 & 100.00 & $<25 \%$ is poor \\
\hline CNS & -2 & 0 & 1 & -1 & 0 & 1 & 0 & -2 to +2 \\
\hline $\mathrm{V}_{\mathrm{RO} 5}$ & 0 & 0 & 0 & 0 & 0 & 0 & 0 & Maximum is 4 \\
\hline $\mathrm{V}_{\mathrm{RO} 3}$ & 0 & 0 & 1 & 0 & 0 & 0 & 0 & Maximum is 3 \\
\hline
\end{tabular}

*Polrz, predicted polarizability in cubic angstroms; $\log \mathrm{P}_{\mathrm{c16}}$, predicted hexadecane/gas partition coefficient; log $\mathrm{P}_{\text {oct, }}$ predicted octanol/gas partition coefficient; $\log \mathrm{P}_{\mathrm{w}}$, predicted water/gas partition coefficient; $\log \mathrm{P}_{\mathrm{o} / \mathrm{w}}$, predicted octanol/water partition coefficient; $\operatorname{logS}$, predicted aqueous solubility; CllogS, conformation-independent predicted aqueous solubility; $\log \mathrm{H}_{\mathrm{ERG}}$, predicted IC $\mathrm{C}_{50}$ value for blockage of HERG $\mathrm{K}^{+}$ channels; PCaco, predicted apparent Caco-2 cell permeability in nm/s; $\operatorname{logBB}$, predicted brain/blood partition coefficient; PMDCK, predicted apparent MDCK cell permeability in $\mathrm{nm} / \mathrm{s} \cdot \log \mathrm{K}_{\mathrm{p}}$, predicted skin permeability; $\log \mathrm{K}_{\mathrm{hsa}}$, prediction of binding to human serum albumin; $\mathrm{pHOA}$, predicted human oral absorption on 0 to $100 \%$ scale; $\mathrm{CNS}$, predicted central nervous sys tem activity on a -2 (inactive) to +2 (active) scale; $\mathrm{V}_{\mathrm{RO} 5}$, 
number of violations of Lipinski's rule of five $(\mathrm{MW}<500, \operatorname{logPo} / \mathrm{w}<5$, donorHB $\leq 5$, accptHB $\leq 10)$; $\mathrm{V}_{\mathrm{RO} 3}$, number of violations of Jorgensen's rule of three (logS $>-5.7$, PCaco $>22$, Primary metabolites $<7$ ).

Furthermore, the following twelve principal descriptors are included in the study: molecular weight (MW), total solvent accessible surface area (SASA), hydrophobic SASA (FOSA), hydrophilic SASA (FISA), carbon Pi SASA (PISA), weakly polar SASA (WPSA), molecular volume (MV), number of rotatable bonds (rotatB), donor-hydrogen bonds (donorHB), acceptor-hydrogen bonds (accptHB), electron affinity $(\mathrm{EA}, \mathrm{eV})$ and ionization potential (IP, eV). Principal descriptors for potential inhibitors and its normal range in $95 \%$ of drugs are given in Figure 5. In the majority of cases, the values of principal descriptors were observed within the normal range for drugs and satisfactory. Therefore, the overall ADMET properties of the selected compounds are found to be suitable as drug candidates.
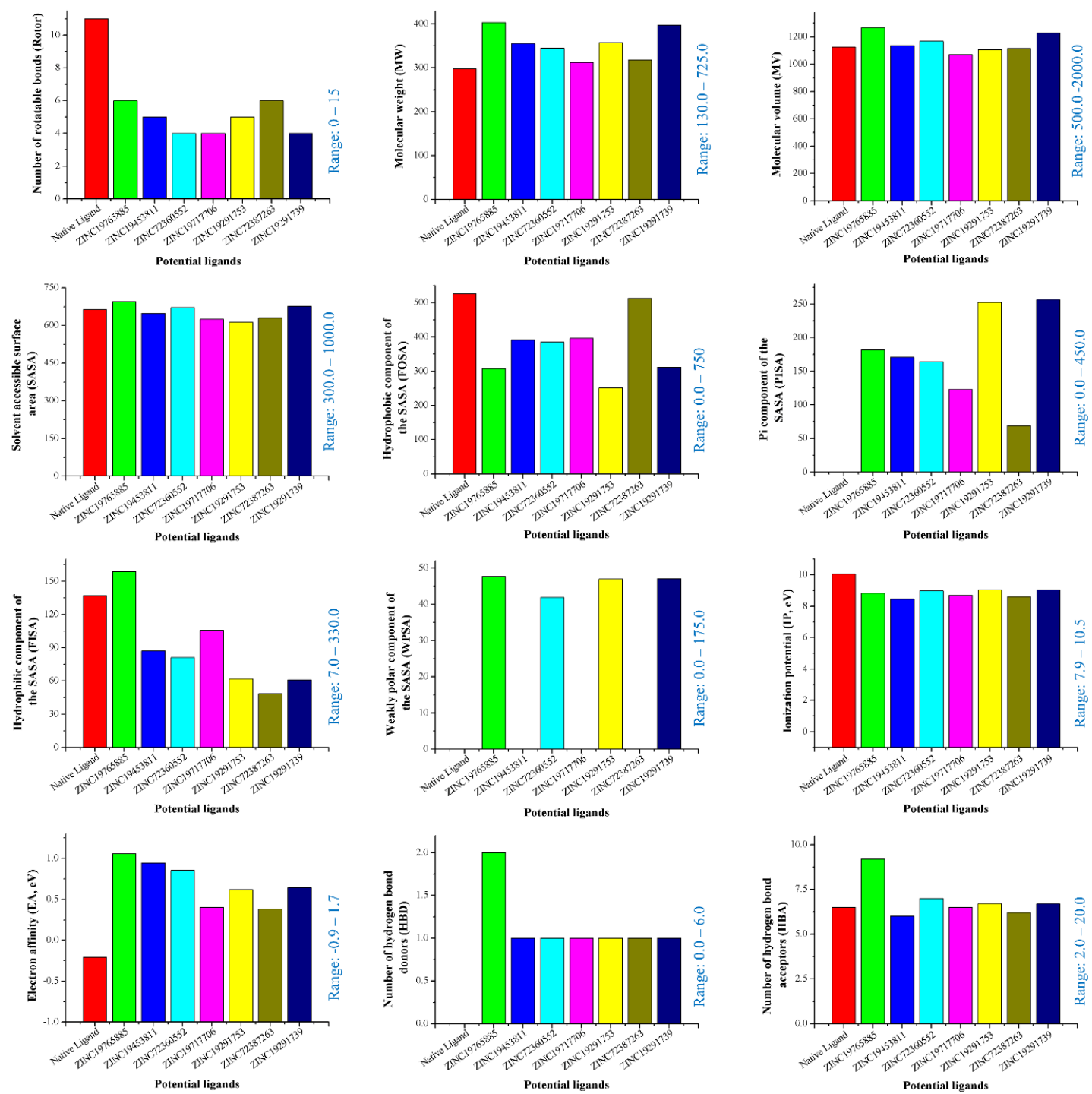

Figure 5: Molecular descriptor profiles of the proposed lead compounds. The range indicated at the right side of each graph is found in $95 \%$ of available drugs. 


\subsection{Post-docking binding energy calculation}

To reassure the molecular docking, the 'pose viewer' files generated in XP docking were submitted to the Prime/MM-GBSA tool for binding free energy calculation. The free energy of binding $\left(\Delta G_{\text {BIND }}\right)$ for the given complexes varied from -128.60 to $-64.17 \mathrm{kcal} / \mathrm{mol}$ (Table 4). Though ZINC19765885 had the lowest Glide score in both SP and XP docking, native ligand performed better in MM-GBSA studies resulting in $-128.60 \mathrm{kcal} / \mathrm{mol}$ than the ZINC19765885 which gave $-68.03 \mathrm{kcal} / \mathrm{mol}$ of free energy. However, the ZINC19765885 was found to be more strained as compared to the native ligand as well as other peers. Among all the potential compounds, the free binding energy of ZINC72387263 was the lowest $(-98.28 \mathrm{kcal} / \mathrm{mol})$ while ZINC19291739 being the highest $(-64.17 \mathrm{kcal} / \mathrm{mol})$. The van der Walls scores of ZINC1976588, ZINC19717706, and ZINC72360552 were closer to that of the native ligand. Therefore, the result indicates that both ZINC72387263 and ZINC19765885 are the best compounds to be the potential QSIs. Since native ligand is not an option here, we only considered the lowest two scorers, ZINC19765885 and ZINC72387263 for MD simulation along with the native ligand as a control.

Table 4: Prime MM-GBSA binding energy calculation of the docked complexes

\begin{tabular}{lllllll}
\hline Compounds & $\Delta \mathbf{G}_{\text {Bind }}$ & $\Delta \mathbf{G}_{\text {Coul }}$ & $\Delta \mathbf{G}_{\text {Lipo }}$ & $\Delta \mathbf{G}_{\mathbf{v d W}}$ & $\Delta \mathbf{G}_{\text {SolvGB }}$ & $\mathbf{S E}_{\text {Lig }}$ \\
\hline Native Ligand & -128.6 & -38.13 & -51.44 & -56.13 & 22.33 & 05.69 \\
ZINC72387263 & -98.28 & -35.92 & -69.39 & -46.49 & 45.41 & 13.32 \\
ZINC19765885 & -96.85 & -16.1 & -63.94 & -55.24 & 23.18 & 26.25 \\
ZINC72360552 & -94.08 & -38.33 & -57.52 & -52.46 & 49.85 & 18.31 \\
ZINC19717706 & -85.79 & -14.48 & -38.4 & -57.71 & 20.78 & 06.23 \\
ZINC19453811 & -74.05 & -9.82 & -47.83 & -40.84 & 19.78 & 17.93 \\
ZINC19291753 & -72.4 & -15.21 & -42.68 & -40.26 & 22.43 & 10.62 \\
ZINC19291739 & -64.17 & 0.82 & -56.85 & -35.61 & 17.01 & 23.93 \\
\hline
\end{tabular}

${ }^{*} \Delta \mathrm{G}_{\text {Bind, }}$ MM-GBSA free binding energy; $\Delta \mathrm{G}_{\mathrm{Coul}}$, Coulomb energy of the complex; $\Delta \mathrm{G}_{\mathrm{vdW}}$, van der Waals energy of the complex; $\Delta \mathrm{G}_{\mathrm{Lipo}}$ lipophilic energy of the complex; $\Delta \mathrm{G}_{\mathrm{SolvGB}}$, solvation energy of the complex; and $\mathrm{SE}_{\mathrm{Lig}}$, strain energy of the ligands.

\subsection{Dynamic simulation studies}

Molecular dynamics simulation (MDS) is essential to determine the stability of the receptor-ligand complex [63]. MD simulation study for $30 \mathrm{~ns}$ time span provides insights into stable molecular interactions between docked protein-ligand complexes under specific physiological circumstances. Figure 5 showed the binding affinity between the receptor and ligand molecules in terms of RMSD and RMSF plots. The energy of the three complexes was fluctuated up to $10 \mathrm{~ns}$. After $10 \mathrm{~ns}$, the stability of the ZINC19765885 and ZINC72387263 was found to be stable throughout the simulation process except at 17 ns where ZINC72387263 showed a little deviation (Figure 6a). The RMSD of ZINC72387263 in the binding pocket during the $10 \mathrm{~ns}$ simulation showed deviations between 1.7 and $2.25 \AA$. The average RMSD values of the ZINC19765885 and ZINC72387263 were $1.6 \AA$ and $1.7 \AA$. On the other hand, the native ligand showed stability at about $5 \mathrm{~ns}$ to $25 \mathrm{~ns}$ after the period it was slightly picked higher and re-stabilized for the rest of the period (Figure 6a). The average RMSD value of the native ligand was $1.2 \AA$. 
The stability of the protein complexes comes from the intra-protein interactions which involve the interfacial residues present in the sidechains [82]. Therefore, the participation of each amino acid residue in the protein-ligand constancy was also analyzed. The fluctuation of the residues was observed to be similar in both leads and native ligands (Figure 6b). RMSF trajectories show that fluctuations of amino residues are found to be higher around the amino residues $0-10,30-45,88-92$, and 160-165. The probable reason of deviation of these amino acids may be a region of the flexible loop and lack of interactions with corresponding ligands [34]. The overall RMSF fluctuations were maintained below $1.0 \AA$, indicating a constant binding of receptor amino acid residues with ligands throughout the simulation time.

(a)

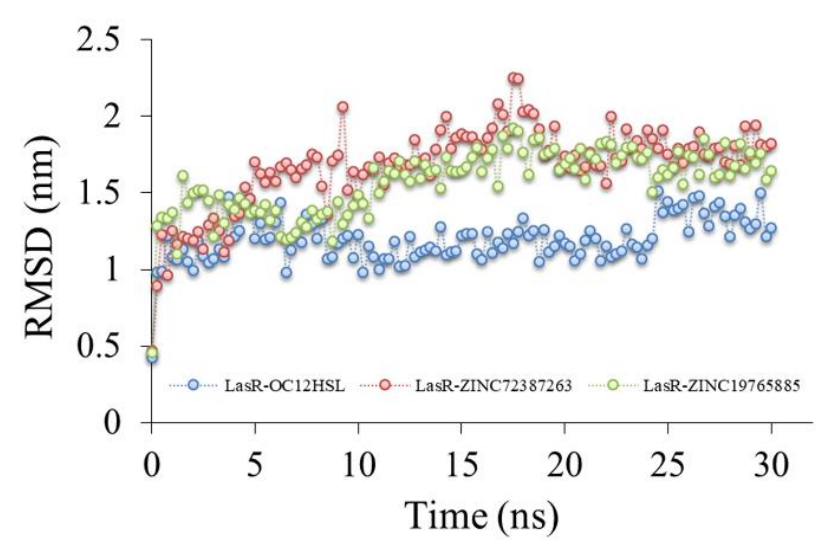

(b)

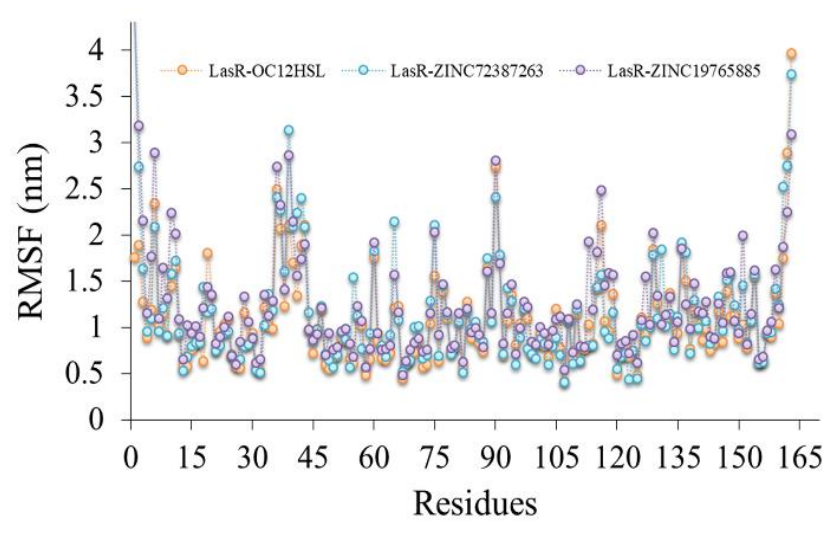

Figure 6: The molecular dynamics simulation of the most promising docked complexes. The studied docked complexes were LasR protein with native and two most potent ligands where (a) root mean square deviation (RMSD) of the protein backbone, (b) root mean square fluctuation (RMSF) of the amino acid residues present in docked complexes.

In average simulation structure, ZINC19765885 and ZINC72387263 showed better interactions than that of the native ligand. All of them maintained six hydrogen bonds on average with a varying number of other non-bonded interactions. The native ligand shared its hydrogen bonds with Ser129, Asp73, and Trp60 residues while ZINC19765885 with Asp73 and Thr75 residues and ZINC72387263 with Asp73 residue. Importantly, the catalytic residue Asp73 maintained hydrogen bonding with all the three ligands. In case of other interactions, ZINC19765885 and ZINC72387263 provided 13 and 8 hydrophobic interactions as compared to the 3 of native ligands. In addition, ZINC19765885 provided single electrostatic contacts and 3 other non-bonded contacts while ZINC72387263 produced an additional saltbridge interaction. The hydrogen bonding and hydrophobic interactions are weak non-bonded interactions which play a key role in stabilizing energetically-favored ligands [83]. Based on the analysis of protein-ligand interactions in PDB database, for example, ligands with high-efficiency are generally enriched with hydrophobic interactions [84]. Therefore, a high number of hydrophobic interactions in the potential QSI candidates may contribute to their efficiency and stability. Moreover, the calculated surface area (SA) of the LasR protein was reduced after binding with inhibitors. For instance, the SA of LasR 
protein was $6798.02 \AA^{2}$ while with the native ligand, ZINC19765885, and ZINC72387263, it reduced to $7270.79,7255.01$ and $7239.03 \AA^{2}$, respectively. This indicates that the ligands were strongly occupying the binding site of the LasR protein. The lesser SA value of ZINC19765885 and ZINC72387263 than the native ligand also suggest their superiority over the native ligand at better fitting.

\section{Conclusion}

In a nutshell, QS in P. aeruginosa works in a hierarchical fashion where attenuation of LasI/R system would shut the whole QS cascade and their functions. We use chemoinformatics approaches to detect potential QSIs from the Asinex Chemical Library. Initially, the high-throughput virtual screening, pharmacophore screening, and molecular docking method recognized seven molecules as potential QSIs. Among them, only two molecules showed good binding energy calculation and ADMET properties closer to the native ligand and selected as the potential QSIs. The binding interaction of the two suggested QSIs with the LasR active site were found stable in molecular dynamic simulation. Therefore, these two QSI compound could potentially inhibit the QS system in P. aeruginosa, thereby, help to reduce the associated complications. Nevertheless, the potentiality of the suggested QSIs needs to be verified by in vitro and in vivo studies as well. Moreover, these finding will help in further drug designing and discovery against $P$. aeruginosa as well as other bacteria that use similar QS system.

\section{Author contribution}

ZN designed and executed the computational works; ZN, SBS, and MAI performed the molecular docking; ZN, UKA, and MMK contributed in writing and critical revision of the manuscript; UKA supervised the whole work. All authors approved the final version of the manuscript for journal submission.

\section{Disclosure statement}

The authors declare no conflict of interest.

\section{ORCID profile}

Zulkar Nain: https:/ /orcid.org/0000-0003-0819-3137

Sifat Bin Sayed: https:/ / orcid.org/0000-0002-4786-0337

Mohammad Minnatul Karim: https://orcid.org/0000-0002-5383-6288

Md. Ariful Islam: https://orcid.org/0000-0003-0102-5587

Utpal Kumar Adhikari: https://orcid.org/0000-0002-0754-8664

\section{References}

[1] G. P. Bodey, R. Bolivar, V. Fainstein, L. Jadeja, Rev Infect Dis 1983, 5, 279.

[2] H. Fazzeli, R. Akbar, S. Moghim, T. Narimani, M. R. Arabestani, A. R. Ghoddousi, J. Res. Med. Sci. 2012, 17, 332.

[3] G. Lila, G. Mulliqi, L. Raka, A. Kurti, R. Bajrami, E. Azizi, Infect. Drug Resist. 2018, 11, 2039.

[4] S. Saxena, G. Banerjee, R. Garg, M. Singh, J. Clin. Diagnostic Res. 2014, 8, 9. 
[5] N. M. Allewell, J. Biol. Chem. 2016, 291, 12527.

[6] N. Høiby, T. Bjarnsholt, M. Givskov, S. Molin, O. Ciofu, Int. J. Antimicrob. Agents 2010, 35, 322.

[7] K. P. Rumbaugh, FEMS Microbiology Letters. Blackwell Publishing Ltd 2014, pp. 141-143.

[8] B. Valot, C. Guyeux, J. Y. Rolland, K. Mazouzi, X. Bertrand, D. Hocquet, PLoS One 2015, $10,1$.

[9] C. L. Ventola, P T A peer-reviewed J. Formul. Manag. 2015, 40, 277.

[10] B. Rémy, S. Mion, L. Plener, M. Elias, E. Chabrière, D. Daudé, Interference in bacterial quorum sensing: A biopharmaceutical perspective. Front. Pharmacol. 2018, 9.

[11] Q. Jiang, J. Chen, C. Yang, Y. Yin, K. Yao, D. Song, Quorum Sensing: A Prospective Therapeutic Target for Bacterial Diseases. Biomed Res. Int. 2019, 2019.

[12] J. P. Pearson, E. C. Pesci, B. H. Iglewski, J. Bacteriol. 1997.

[13] T. R. Kievit, Y. Kakai, J. K. Register, E. C. Pesci, B. H. Iglewski, FEMS Microbiol. Lett. 2002, 212, 101.

[14] M. Kalia, P. K. Singh, V. K. Yadav, B. S. Yadav, D. Sharma, S. S. Narvi, et al., Microb. Pathog. 2017, 107, 136.

[15] S. Y. Y. Tan, S. L. Chua, Y. Chen, S. A. Rice, S. Kjelleberg, T. E. Nielsen, et al., Antimicrob. Agents Chemother. 2013, 57, 5629.

[16] M. V. Turkina, E. Vikström, Bacteria-Host Crosstalk: Sensing of the Quorum in the Context of Pseudomonas aeruginosa Infections. J. Innate Immun. 2019, 11, 263-279.

[17] S. T. Rutherford, B. L. Bassler, Cold Spring Harb. Perspect. Med. 2012, 2, a012427.

[18] C. T. O'Loughlin, L. C. Miller, A. Siryaporn, K. Drescher, M. F. Semmelhack, B. L. Bassler, Proc. Natl. Acad. Sci. 2013, 110, 17981.

[19] T. B. Rasmussen, M. E. Skindersoe, T. Bjarnsholt, R. K. Phipps, K. B. Christensen, P. O. Jensen, et al., Microbiology 2005, 151, 1325.

[20] T. H. Jakobsen, M. Van Gennip, R. K. Phipps, M. S. Shanmugham, L. D. Christensen, M. Alhede, et al., Antimicrob. Agents Chemother. 2012, 56, 2314.

[21] S. Sarabhai, K. Harjai, P. Sharma, N. Capalash, J. Appl. Microbiol. 2015, 118, 817.

[22] C.-L. Koh, C.-K. Sam, W.-F. Yin, L. Y. Tan, T. Krishnan, Y. M. Chong, et al., Sensors (Basel). 2013, 13, 6217.

[23] M. Hentzer, M. Givskov, J. Clin. Invest. 2003, 112, 1300.

[24] A. Algburi, S. Zehm, V. Netrebov, A. B. Bren, V. Chistyakov, M. L. Chikindas, Probiotics Antimicrob. Proteins 2017, 9, 81.

[25] C. E. Shelburne, F. Y. An, V. Dholpe, A. Ramamoorthy, D. E. Lopatin, M. S. Lantz, J. Antimicrob. Chemother. 2007, 59, 297.

[26] C. Luise, D. Robaa, Methods Mol. Biol. 2018, 1824, 347.

[27] E. Lionta, G. Spyrou, D. Vassilatis, Z. Cournia, Curr. Top. Med. Chem. 2014, 14, 1923. 
[28] B. Waszkowycz, Curr. Opin. Drug Discov. Devel. 2002, 5, 407.

[29] L. Yang, M. T. Rybtke, T. H. Jakobsen, M. Hentzer, T. Bjarnsholt, M. Givskov, et al., Antimicrob. Agents Chemother. 2009, 53, 2432.

[30] S. Grüneberg, B. Wendt, G. Klebe, Angew. Chem. Int. Ed. Engl. 2001, 40, 389.

[31] T. N. Doman, S. L. McGovern, B. J. Witherbee, T. P. Kasten, R. Kurumbail, W. C. Stallings, et al., J. Med. Chem. 2002, 45, 2213.

[32] H. J. Boehm, M. Boehringer, D. Bur, H. Gmuender, W. Huber, W. Klaus, et al., J. Med. Chem. 2000, 43, 2664.

[33] S. Ghosh, A. Nie, J. An, Z. Huang, Curr. Opin. Chem. Biol. 2006, 10, 194.

[34] P. Natarajan, V. Priyadarshini, D. Pradhan, M. Manne, S. Swargam, H. Kanipakam, et al., J. Recept. Signal Transduct. 2016, 36, 445.

[35] Hymavati, V. Kumar, M. Elizabeth Sobhia, Comput. Math. Methods Med. 2012, 2012.

[36] G. A. Kaminski, R. A. Friesner, J. Tirado-Rives, W. L. Jorgensen, J. Phys. Chem. B 2001, 105, 6474.

[37] D. Shivakumar, J. Williams, Y. Wu, W. Damm, J. Shelley, W. Sherman, J. Chem. Theory Comput. 2010, 6, 1509.

[38] R. A. Friesner, J. L. Banks, R. B. Murphy, T. A. Halgren, J. J. Klicic, D. T. Mainz, et al., J. Med. Chem. 2004, 47, 1739.

[39] T. A. Halgren, R. B. Murphy, R. A. Friesner, H. S. Beard, L. L. Frye, W. T. Pollard, et al., J. Med. Chem. 2004, 47, 1750.

[40] J. J. Irwin, T. Sterling, M. M. Mysinger, E. S. Bolstad, R. G. Coleman, ZINC: A free tool to discover chemistry for biology. J. Chem. Inf. Model. 2012, 52, 1757-1768.

[41] J. C. Shelley, A. Cholleti, L. L. Frye, J. R. Greenwood, M. R. Timlin, M. Uchimaya, J. Comput. Aided. Mol. Des. 2007, 21, 681.

[42] C. A. Lipinski, B. W. Dominy, P. J. Feeney, Adv. Drug Deliv. Rev. 1997.

[43] G. M. Rishton, Reactive compounds and in vitro false positives in HTS. Drug Discov. Today 1997, 2, 382-384.

[44] M. Karthikeyan, R. Vyas, Practical chemoinformatics, 2014.

[45] A. Dhasmana, S. Raza, R. Jahan, M. Lohani, J. M. Arif, In New Look to Phytomedicine, Elsevier, 2019, pp. 523-548.

[46] S. Subramaniam, M. Mehrotra, D. Gupta, Bioinformation 2008, 3, 14.

[47] N. K. Salam, R. Nuti, W. Sherman, J. Chem. Inf. Model. 2009, 49, 2356.

[48] G. K. Veeramachaneni, K. K. Raj, L. M. Chalasani, J. S. Bondili, V. R. Talluri, Drug Des. Devel. Ther. 2015, 9, 4397.

[49] S. Kim, P. A. Thiessen, E. E. Bolton, J. Chen, G. Fu, A. Gindulyte, et al., Nucleic Acids Res. 2016, 44, D1202.

[50] R. A. Friesner, R. B. Murphy, M. P. Repasky, L. L. Frye, J. R. Greenwood, T. A. Halgren, et 
al., J. Med. Chem. 2006, 49, 6177.

[51] Z. Chen, G. Tian, Z. Wang, H. Jiang, J. Shen, W. Zhu, J. Chem. Inf. Model. 2010, 50, 615.

[52] R. Palakurti, D. Sriram, P. Yogeeswari, R. Vadrevu, Mol. Inform. 2013, 32, 385.

[53] T. Kaserer, K. R. Beck, M. Akram, A. Odermatt, D. Schuster, P. Willett, Pharmacophore models and pharmacophore-based virtual screening: Concepts and applications exemplified on hydroxysteroid dehydrogenases. Molecules 2015, 20, 22799-22832.

[54] N. Triballeau, F. Acher, I. Brabet, J. P. Pin, H. O. Bertrand, J. Med. Chem. 2005, 48, 2534.

[55] J. F. Truchon, C. I. Bayly, J. Chem. Inf. Model. 2007, 47, 488.

[56] R. P. Sheridan, S. B. Singh, E. M. Fluder, S. K. Kearsley, J. Chem. Inf. Comput. Sci. 2001, 41, 1395.

[57] C. Empereur-Mot, H. Guillemain, A. Latouche, J. F. Zagury, V. Viallon, M. Montes, J. Cheminform. 2015, 7.

[58] T. A. Halgren, R. B. Murphy, R. A. Friesner, H. S. Beard, L. L. Frye, W. T. Pollard, et al., J. Med. Chem. 2004, 47, 1750.

[59] D. Toledo Warshaviak, G. Golan, K. W. Borrelli, K. Zhu, O. Kalid, J. Chem. Inf. Model. 2014, $54,1941$.

[60] S. L. Dixon, A. M. Smondyrev, S. N. Rao, PHASE: A novel approach to pharmacophore modeling and 3D database searching. Chem. Biol. Drug Des. 2006, 67, 370-372.

[61] K. Muthusamy, K. D. Singh, S. Chinnasamy, S. Nagamani, G. Krishnasamy, C. Thiyagarajan, et al., Interdiscip. Sci. Comput. Life Sci. 2013, 5, 119.

[62] N. Kandakatla, G. Ramakrishnan, Adv. Bioinformatics 2014, 2014.

[63] S. Borkotoky, C. K. Meena, A. Murali, Bioinform. Biol. Insights 2016, 10, 155.

[64] D. Das, Y. Koh, Y. Tojo, A. K. Ghosh, H. Mitsuya, J. Chem. Inf. Model. 2009, 49, 2851.

[65] T. Hou, J. Wang, Y. Li, W. Wang, J. Chem. Inf. Model. 2011, 51, 69.

[66] B. Vijayakumar, S. Parasuraman, R. Raveendran, D. Velmurugan, Pharmacogn. Mag. 2014, 10, S639.

[67] P. D. Lyne, M. L. Lamb, J. C. Saeh, J. Med. Chem. 2006, 49, 4805.

[68] S. Saxena, L. Durgam, L. Guruprasad, J. Biomol. Struct. Dyn. 2019, 37, 1783.

[69] L. Ioakimidis, L. Thoukydidis, A. Mirza, S. Naeem, J. Reynisson, QSAR Comb. Sci. 2008, 27,445 .

[70] K. Liu, H. Kokubo, J. Chem. Inf. Model. 2017, 57, 2514.

[71] C. J. Dickson, B. D. Madej, Å. A. Skjevik, R. M. Betz, K. Teigen, I. R. Gould, et al., J. Chem. Theory Comput. 2014, 10, 865.

[72] E. Krieger, T. Darden, S. B. Nabuurs, A. Finkelstein, G. Vriend, Proteins Struct. Funct. Bioinforma. 2004, 57, 678.

[73] S. Qiu, L. M. Azofra, D. R. MacFarlane, C. Sun, Phys. Chem. Chem. Phys. 2018, 20, 6735.

[74] P. a Sigala, M. a Tsuchida, D. Herschlag, Proc. Natl. Acad. Sci. 2009, 106, 9232. 
[75] P. Kiratisin, K. D. Tucker, L. Passador, J. Bacteriol. 2002, 184, 4912.

[76] H. M. Berman, Nucleic Acids Res. 2000, 28, 235.

[77] R. E. Hubbard, M. Kamran Haider, In Encyclopedia of Life Sciences, John Wiley \& Sons, Ltd, Chichester, UK, 2010.

[78] A. C. Tripathi, P. K. Sonar, R. Rathore, S. K. Saraf, Open Pharm. Sci. J. 2016, 3, 164.

[79] M. Ahumedo, M. Velásquez, R. Vivas Reyes, Rev. Vitae 2017, 24, 89.

[80] O. Dror, D. Schneidman-Duhovny, Y. Inbar, R. Nussinov, H. J. Wolfson, J. Chem. Inf. Model. 2009, 49, 2333.

[81] M. Congreve, R. Carr, C. Murray, H. Jhoti, A "Rule of Three" for fragment-based lead discovery? Drug Discov. Today 2003, 8, 876-877.

[82] S. Jayashree, P. Murugavel, R. Sowdhamini, N. Srinivasan, Biol. Direct 2019, 14.

[83] R. Patil, S. Das, A. Stanley, L. Yadav, A. Sudhakar, A. K. Varma, PLoS One 2010, 5, e12029.

[84] R. Ferreira De Freitas, M. Schapira, Medchemcomm 2017, 8, 1970. 